\title{
Modeling brain dynamics after tumor resection using The Virtual Brain
}

\author{
Hannelore Aerts ${ }^{\mathrm{a}}$, Michael Schirner ${ }^{\mathrm{b}, \mathrm{c}}$, Thijs Dhollander ${ }^{\mathrm{d}, \mathrm{e}}$, Ben Jeurissen ${ }^{\mathrm{f}}$, Eric Achten ${ }^{\mathrm{g}}$, \\ Dirk Van Roost ${ }^{\mathrm{h}}$, Petra Ritter ${ }^{\mathrm{b}, \mathrm{c}}$, Daniele Marinazzo ${ }^{\mathrm{a}, *}$ \\ ${ }^{a}$ Department of Data-Analysis, Faculty of Psychology and Educational Sciences, Ghent University, Belgium \\ ${ }^{\mathrm{b}}$ Charité - Universitätsmedizin Berlin, Corporate Member of Freie Universität Berlin, Humboldt-Universität zu Berlin, And Berlin Institute of Health, Dept. of Neurology, \\ Germany \\ ${ }^{\mathrm{c}}$ Bernstein Focus State Dependencies of Learning \& Bernstein Center for Computational Neuroscience, Berlin, Germany \\ d The Florey Institute of Neuroscience and Mental Health, Melbourne, Australia \\ e The Florey Department of Neuroscience and Mental Health, University of Melbourne, Australia \\ ${ }^{\mathrm{f}}$ Imec - Vision Lab, Department of Physics, University of Antwerp, Belgium \\ ${ }^{g}$ Department of Neuroradiology, Ghent University Hospital, Ghent, Belgium \\ ${ }^{\mathrm{h}}$ Department of Neurosurgery, Ghent University Hospital, Belgium
}

\section{A R T I C L E I N F O}

\section{Keywords:}

Brain tumor

Computational neuroscience

Connectome

Functional connectivity

Graph theory

Neuroinformatics

\begin{abstract}
A B S T R A C T
Brain tumor patients scheduled for tumor resection often face significant uncertainty, as the outcome of neurosurgery is difficult to predict at the individual patient level. Recently, simulation of the activity of neural populations connected according to the white matter fibers, producing personalized brain network models, has been introduced as a promising tool for this purpose. The Virtual Brain provides a robust open source framework to implement these models. However, brain network models first have to be validated, before they can be used to predict brain dynamics. In prior work, we optimized individual brain network model parameters to maximize the fit with empirical brain activity. In this study, we extend this line of research by examining the stability of fitted parameters before and after tumor resection, and compare it with baseline parameter variability using data from healthy control subjects. Based on these findings, we perform the first "virtual neurosurgery", mimicking patient's actual surgery by removing white matter fibers in the resection mask and simulating again neural activity on this new connectome.

We find that brain network model parameters are relatively stable over time in brain tumor patients who underwent tumor resection, compared with baseline variability in healthy control subjects. Concerning the virtual neurosurgery analyses, use of the pre-surgery model implemented on the virtually resected structural connectome resulted in improved similarity with post-surgical empirical functional connectivity in some patients, but negligible improvement in others. These findings reveal interesting avenues for increasing interactions between computational neuroscience and neuro-oncology, as well as important limitations that warrant further investigation.
\end{abstract}

\section{Introduction}

Many brain tumor patients undergoing neurosurgery face significant uncertainty regarding the outcome of surgery. Average neurosurgical outcomes for patient cohorts can be predicted with a varying degree of accuracy (Emblem et al., 2015; Senders et al., 2017); the heterogeneity of brain tumors complicates predictions on an individual patient level.

Following methodological advances, several studies have addressed this limitation by applying graph theoretical and machine learning approaches to infer neurosurgical outcome at the individual patient level (for a review see Senders et al., 2018). In particular, several studies have tried to find biomarkers that predict seizure freedom after epilepsy surgery (for example Bonilha et al., 2013, 2015; He et al., 2017; Ji et al., 2015; Morgan et al., 2017; Munsell et al., 2015; Taylor et al., 2018; van Dellen et al., 2014). Others have evaluated machine learning strategies designed to predict survival in glioma (Emblem et al., 2009, 2015) and traumatic brain injury patients (Rughani et al., 2010). Furthermore, one study found that graph measures derived from the pre-surgical functional connectome of patients with temporal lobe epilepsy were able to predict post-surgical cognitive performance scores across different domains (Doucet et al., 2015).

\footnotetext{
* Corresponding author. Henri Dunantlaan 2, 9000 Gent, Belgium.

E-mail address: daniele.marinazzo@ugent.be (D. Marinazzo).
} 
Recently, brain network modeling has also been introduced as a promising tool to simulate neurosurgical outcome (Arsiwalla et al., 2015; Proix et al., 2017). Brain network modeling techniques implement dynamical models on individual structural brain connectivity networks to simulate subject-specific brain activity (Schirner et al., 2018). By virtually lesioning structural connectomes, brain network models may therefore be used as predictive tools to investigate the impact of diverse structural connectivity alterations on brain dynamics, including those purposefully induced by surgery.

For example, a study by Sinha and colleagues (Sinha et al., 2017) investigated surgical outcome in patients undergoing neurosurgery for refractory epilepsy. Specifically, they modeled seizure likelihood per region to identify a highly epileptogenic zone in each patient. According to model predictions, virtual resection of these regions with high seizure likelihood reduced the overall likelihood of seizures, which was confirmed by actual surgical outcomes in the majority of patients (81.3\%). Moreover, in patients with poor predicted outcomes, alternative resection sites could be obtained from the model. Furthermore, it has been shown that large-scale brain network models can be used to predict the propagation zone of epileptic activity as determined by stereotactic EEG recordings and clinical expertise (Proix et al., 2017). Importantly, in a follow-up study, they were able to identify the most unstable pathways that support and allow the propagation of seizure activity (Olmi et al., 2019). Hence, results from this study suggest that selective removal of these unstable connections would be equally effective to render patients seizure free, compared with surgical resection of the entire epileptogenic zone.

The major advantage of brain network modeling is that it produces actual biophysically-oriented models of the brain that go beyond a simple black-box predictor of surgical outcome, potentially making it a useful tool to predict a rich variety of outcomes such as epilepsy status, cognitive performance, functional network integrity and survival. Brain network modeling may thus serve as an important complementary source of information to aid patients and physicians in the process of surgical and medical decision making, by providing estimates of successful and/ or adverse outcomes. Furthermore, biologically inspired dynamical models may provide insights into the local dynamics underlying largescale network topology in health and disease. As such, they may provide an entry point for understanding brain disorders as well as recovery processes after interventions at a causal mechanistic level.

In prior work (Aerts et al., 2018) we investigated brain dynamics before tumor resection in 25 brain tumor patients and 11 healthy control subjects using The Virtual Brain (TVB) (Sanz Leon et al., 2013); an open-source neuroinformatics platform that enables the construction, simulation and analysis of large-scale brain network models. In particular, we optimized model parameters of the Reduced Wong-Wang model (Deco et al., 2014) on an individual basis, after which we compared the fitted parameters between brain tumor patients and healthy control subjects. In addition, we assessed the relations between model parameters, structural network topology and cognitive performance. We found significantly improved prediction accuracy of individual functional connectivity when using individually optimized model parameters, indicating the importance of tuning the model parameters in a subject-specific manner. In addition, local model parameters differed between regions directly affected by a tumor, regions distant from a tumor, and regions in a healthy brain. Lastly, we set to identify several associations between model parameters, structural network topology and cognitive performance.

In this study, we extend this line of research by examining possible changes in optimized model parameters from pre-to post-operative assessment. To this end, we apply the same procedure as in the preoperative case to the data acquired several months after each patient's surgery. To quantify a normal range of baseline variability over time, we also perform parameter optimization on data acquired from healthy control subjects at both time points. After examining the stability of fitted model parameters over time, we use this information to perform the first "virtual neurosurgery" analyses on glioma patients' pre-operative data, to evaluate the potential of brain network modeling to predict brain dynamics after tumor resection. The workflow of the procedure is sketched in Fig. 1.

\section{Methods}

\subsection{Participants}

Patients for this study were recruited with the aim of longitudinal assessment. In particular, data were collected the day before each patient's tumor resection and again several months after surgery, on the day of patients' first clinical consultation at the hospital (mean: 7.9 months post-operative; range $5.2-10.7$ months post-operative). Patients were included if they were diagnosed with a glioma or meningioma (Fisher et al., 2007). Both types of tumors are typically graded according to their malignancy, with grade I tumors being benign, and grade III (for meningioma) or IV (for glioma) being most malignant (Louis et al., 2007). Hereby, malignancy depends on the speed with which the disease evolves, the extent to which the tumor infiltrates healthy brain tissue, and chances of recurrence or progression to higher grades of malignancy.

Patients were recruited from Ghent University Hospital (Belgium) between May 2015 and October 2017. Patients were eligible if they (1) were at least 18 years old, (2) had a supratentorial meningioma (WHO grade I or II) or glioma (WHO grade II or III), (3) were able to complete neuropsychological testing, and (4) were medically approved to undergo MRI investigation. Primary caregivers of the patients were also asked to participate in the study to constitute a group of healthy control subjects that suffer from comparable emotional distress as the patients (Goebel et al., 2011; Janda et al., 2007). All participants received detailed study information and gave written informed consent prior to study enrollment. This study was approved by the Ethics Committee of Ghent University Hospital.

Out of the 11 glioma patients (mean age $47.5 \mathrm{y}, S D=11.3 \mathrm{y} ; 36 \%$ females), 14 meningioma patients (mean age 60.4y, $S D=12.3 \mathrm{y} ; 79 \%$ females) and 11 healthy controls (mean age 58.6y, $S D=10.3 \mathrm{y} ; 36 \%$ females; 10 spouses, 1 adult child) that were included pre-surgically, 7 glioma patients (mean age pre-operatively $50.7 \mathrm{y}, S D=11.7 \mathrm{y} ; 43 \%$ females), 11 meningioma patients (mean age pre-operatively $57.9 \mathrm{y}, S D=$ $11.0 \mathrm{y} ; 80 \%$ females), and 10 control subjects (mean age pre-operatively 59.6, $S D=10.3 \mathrm{y} ; 40 \%$ females; 9 spouses, 1 adult child) agreed to participate post-operatively. Patient characteristics including follow-up information are described in Table 1.

\subsection{MRI data acquisition and preprocessing}

MRI sequence details and preprocessing procedures for the postoperative data are mostly identical to those that we used before to collect and preprocess the pre-operative data. All details are described in Aerts et al. (2018). In the following sections, we provide a summary of these procedures, as well as an overview of the minor modifications that were applied.

\subsubsection{MRI data acquisition}

From all participants, three types of MRI scans were obtained using a Siemens 3T Magnetom Trio MRI scanner with a 32-channel head coil. First, a T1-weighted MPRAGE image was acquired (160 slices, TR = $1750 \mathrm{~ms}, \mathrm{TE}=4.18 \mathrm{~ms}$, field of view $=256 \mathrm{~mm}$, flip angle $=9^{\circ}$, voxel size $1 \times 1 \times 1 \mathrm{~mm}$, acquisition time of 4:05 min). Next, resting-state functional echo-planar imaging (EPI) data were obtained in an 


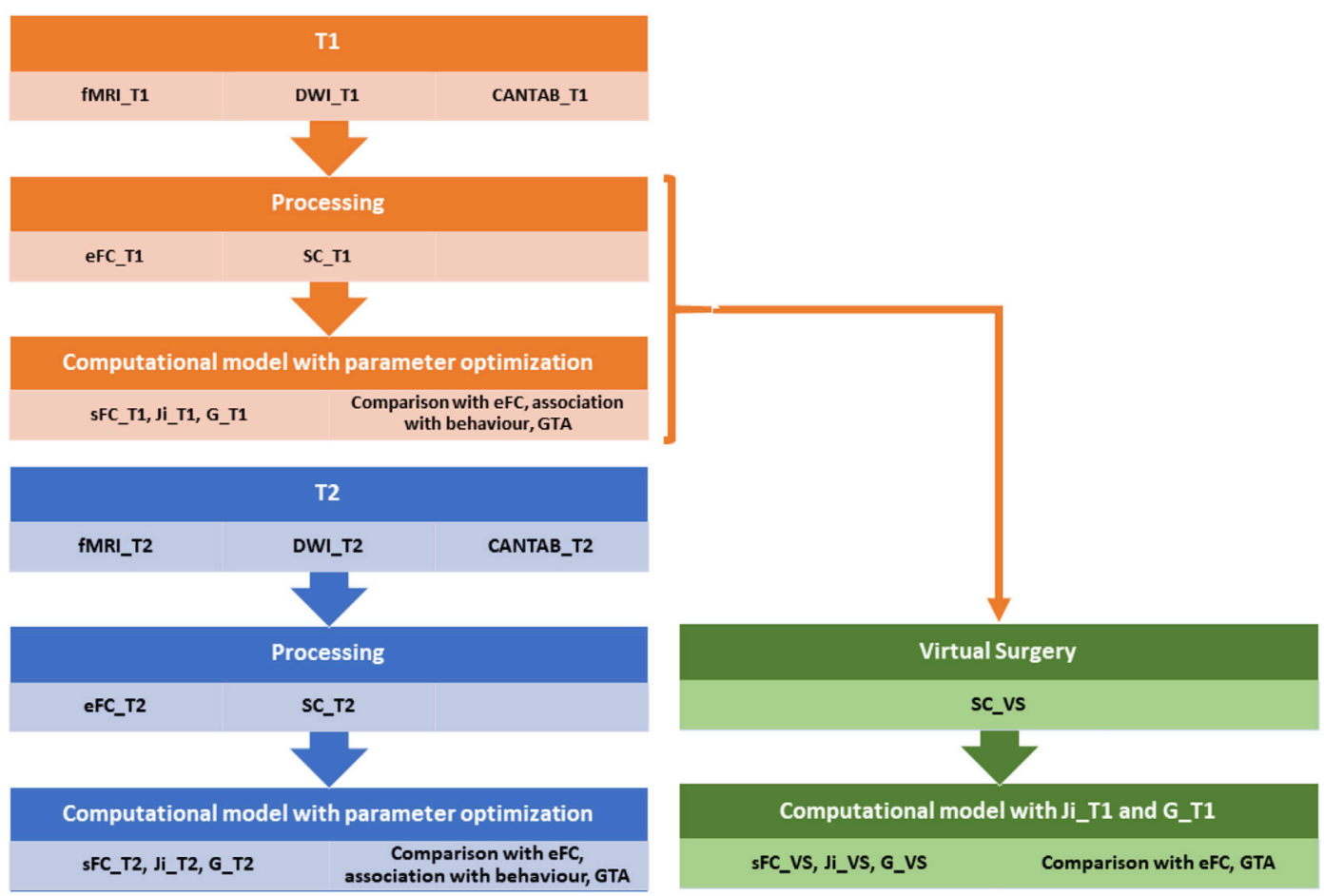

Fig. 1. Workflow of the procedure. Data (fMRI, DWI, behavioral) from brain tumor patients and controls are acquired at two times: T1 (pre-surgery) and T2 (postsurgery). Empirical correlation matrix of activity (eFC) and structural connectivity (SC) are derived from fMRI and DWI data respectively. In both cases The Virtual Brain is used to build personalized models of brain activity, connecting models of neural populations according to SC, and optimizing model parameters to maximize similarity between the simulated pattern of correlated activity $(\mathrm{sFC})$ and eFC. The model parameters are associated to behavioral measures and measures derived from Graph Theory Analysis (GTA). Additionally Virtual Surgery (VS) is performed using data and model parameters from T1, according to the procedure detailed below.

interleaved order (42 slices, $\mathrm{TR}=2100 \mathrm{~ms}, \mathrm{TE}=27 \mathrm{~ms}$, field of view $=$ $192 \mathrm{~mm}$, flip angle $=90^{\circ}$, voxel size $3 \times 3 \times 3 \mathrm{~mm}$, acquisition time of 6:24 min). ${ }^{1}$ During the fMRI scan, participants were instructed to keep their eyes closed and not fall asleep. Finally, multi-shell high-angular resolution diffusion imaging (HARDI) MRI data were acquired (60 slices, $\mathrm{TR}=8700 \mathrm{~ms}, \mathrm{TE}=110 \mathrm{~ms}$, field of view $=240 \mathrm{~mm}, 102$ gradient directions, b-values of $0,700,1200,2800 \mathrm{~s} / \mathrm{mm}^{2}$, voxel size 2.5 x $2.5 \times 2.5$ $\mathrm{mm}$, acquisition time of 15:14 min) (Jeurissen et al., 2014). In addition, two diffusion MRI b $=0 \mathrm{~s} / \mathrm{mm}^{2}$ images were collected with reversed phase-encoding blips to correct susceptibility induced distortions (Andersson et al., 2003).

\subsubsection{Preprocessing of T1-weighted anatomical MRI data}

High-resolution anatomical images were processed using the default "recon-all" processing pipeline of FreeSurfer (http://surfer.nmr.mgh.ha rvard.edu), yielding a subject-specific parcellation of each participant's cortex into 68 regions (Desikan et al., 2006; Fischl et al., 2004). To account for lesion effects in the parcellation, some additional steps were performed depending on the specific tumor type. For meningioma tumors, that were completely resected during neurosurgery, few to no lesion effects were apparent in eight out of ten patients. For these patients, the default processing pipeline was applied. In the other two meningioma patients, residual edema and a resection cavity were observed, respectively. Therefore, we used a procedure similar to the one outlined in Solodkin et al. (2010). Specifically, we first produced an enantiomorphic filling of the lesioned area (Nachev et al., 2008) using

\footnotetext{
${ }^{1}$ After the first patient was tested for follow-up, the resting-state fMRI protocol was accidentally changed from a TR of $2100 \mathrm{~ms}$ to a TR of $2400 \mathrm{~ms}$ resulting in a slightly longer acquisition time of 7:19 min. In prior work, we have however demonstrated that such slight alterations in TR have little impact on the construction of FC matrices or the model parameter optimization (Aerts et al., 2018).
}

the BCBtoolkit (Foulon et al., 2018), after which the standard FreeSurfer processing pipeline was utilized. For the glioma patients, we used each patient's pre-operative parcellation scheme, after non-linear registration to their post-operative space (using FSL FNIRT; Andersson et al., 2007). All registration results were visually verified.

\subsubsection{Functional MRI preprocessing}

Resting-state fMRI data were preprocessed using FEAT (FMRI Expert Analysis Tool, version 6.00), part of FSL (FMRIB's Software Library; Jenkinson, Beckmann, Behrens, Woolrich and Smith, 2012), comprising motion correction, slice-timing correction, non-brain removal, grand-mean intensity normalization and high-pass temporal filtering (100-s high-pass filter). Functional connectivity matrices were then constructed by mapping the FreeSurfer cortical parcellation schemes obtained in the previous step to each subject's functional MRI data, and calculating the Fisher's z-transformed Pearson correlation coefficient between all region-wise BOLD time series.

\subsubsection{Diffusion MRI preprocessing}

For preprocessing and construction of structural connectomes based on the diffusion MRI (dMRI) data, a processing pipeline was used combining FSL (FMRIB's Software Library; Jenkinson et al., 2012; version 5.0.9) and MRtrix3 (Tournier et al., 2019). Preprocessing steps included correction for various artifacts (noise (Veraart et al., 2016), Gibbs ringing (Kellner et al., 2016), motion and eddy currents (Andersson and Sotiropoulos, 2016), susceptibility induced distortions (Andersson et al., 2003) and bias field inhomogeneities (Zhang et al., 2001)), registration of subjects' high-resolution anatomical images to diffusion space (Jenkinson et al., 2002; Jenkinson and Smith, 2001), and segmentation of the anatomical images into gray matter, white matter and cerebrospinal fluid (Zhang et al., 2001). Further, quantitative whole-brain probabilistic tractography was performed using MRtrix3 (Tournier et al., 2019), resulting in 7.5 million streamlines per subject 
Table 1

Patient characteristics. Gray shading indicates the patients included in the Virtual Surgery.

\begin{tabular}{|c|c|c|c|c|c|c|c|c|}
\hline $\begin{array}{c}\text { Subject } \\
\text { ID }\end{array}$ & Sex & Age $^{1}$ & Handedness $^{1,2}$ & $\begin{array}{c}\text { Tumor } \\
\text { lateralization }\end{array}$ & $\begin{array}{c}\text { Tumor } \\
\text { location }\end{array}$ & $\begin{array}{c}\text { Tumor } \\
\text { size }\left(\mathrm{cm}^{3}\right) \\
\end{array}$ & Tumor histology ${ }^{3}$ & $\begin{array}{c}\text { T2-T1 interval } \\
\text { (days) }\end{array}$ \\
\hline CON01 & $M$ & 49 & -1.00 & & & & & \\
\hline CONO2 & $M$ & 64 & 1.00 & & & & & \\
\hline CONO3 & $\mathrm{F}$ & 53 & 1.00 & & & & & \\
\hline CONO4 & $\mathrm{F}$ & 53 & 1.00 & & & & & \\
\hline CON05 & M & 49 & 1.00 & & & & & \\
\hline CON06 & M & 72 & 1.00 & & & & & \\
\hline CONO7 & $\mathrm{F}$ & 48 & -0.20 & & & & & \\
\hline CON08 & M & 75 & 1.00 & & & & & \\
\hline CONO9 & $M$ & 58 & 1.00 & & & & & \\
\hline CON10 & $\mathrm{M}$ & 52 & 0.90 & & & & & \\
\hline CoN11 & $\mathrm{F}$ & 72 & 1.00 & & & & & \\
\hline PAT01 & $\mathrm{F}$ & 67 & 1.00 & Left & Frontal & 1.69 & Meningioma I & 182 \\
\hline PAT02 & $\mathrm{F}$ & 49 & 1.00 & Right & Frontal & 0.75 & Meningioma I & 238 \\
\hline РАT03 & $\mathrm{F}$ & 60 & 1.00 & Right & Parietal & 78.44 & Meningioma I & 192 \\
\hline PAT05 & $\mathrm{F}$ & 40 & 1.00 & Left & Frontal & 12.95 & Oligo-astrocytoma II & 144 \\
\hline РAT06 & $\mathrm{F}$ & 67 & 1.00 & Bilateral & Frontal & 12.81 & Meningioma I & 260 \\
\hline PAT07 & $\mathrm{M}$ & 55 & -0.16 & Left & Temporal & 33.94 & Ependymoma II & 227 \\
\hline РАT08 & $M$ & 51 & 1.00 & Left & Frontal & 16.81 & Meningioma I & 308 \\
\hline PAT10 & $\mathrm{F}$ & 77 & 1.00 & Left & Frontal & 0.59 & Meningioma I & 270 \\
\hline PAT11 & $\mathrm{F}$ & 74 & 1.00 & Left & Frontal & 20.73 & Meningioma II & $\begin{array}{l}\text { Insufficient } \\
\text { data quality }\end{array}$ \\
\hline PAT13 & $\mathrm{F}$ & 40 & -0.68 & Bilateral & Skull base & 2.09 & Meningioma I & 261 \\
\hline PAT14 & $\mathrm{F}$ & 74 & 1.00 & Left & Parietal & 3.45 & Meningioma I & MRI impossible \\
\hline PAT15 & $\mathrm{F}$ & 57 & 1.00 & Right & Frontal & 2.13 & Meningioma I & 212 \\
\hline PAT16 & M & 39 & 1.00 & Right & Fronto-temporal & 50.24 & $\begin{array}{c}\text { Anaplastic } \\
\text { astrocytoma II-III }\end{array}$ & 246 \\
\hline PAT17 & $\mathrm{F}$ & 49 & 1.00 & Right & Frontal & 0.58 & Meningioma I & 296 \\
\hline PAT19 & $\mathrm{F}$ & 44 & 1.00 & Right & $\begin{array}{c}\text { Frontal skull } \\
\text { base }\end{array}$ & 2.64 & Meningioma I & Drop-out \\
\hline PAT20 & $\mathrm{F}$ & 70 & 0.60 & Right & Parietal & 15.24 & $\begin{array}{c}\text { Anaplastic } \\
\text { astrocytoma III }\end{array}$ & 184 \\
\hline PAT22 & M & 53 & 1.00 & Right & Parietal & 15.16 & Oligodendroglioma II & No resection \\
\hline РAT23 & $\mathrm{M}$ & 74 & 1.00 & Bilateral & Frontal & 89.56 & Meningioma I & 251 \\
\hline PAT24 & $\mathrm{M}$ & 62 & 1.00 & Left & Frontal & 5.91 & Meningioma I & 203 \\
\hline PAT25 & $\mathrm{F}$ & 46 & 1.00 & Right & Temporal & 18.56 & Glioma II & 258 \\
\hline РAT26 & M & 61 & 1.00 & Right & Temporal & 59.21 & $\begin{array}{c}\text { Anaplastic } \\
\text { astrocytoma III }\end{array}$ & 259 \\
\hline РAT27 & M & 38 & 1.00 & Left & Frontal & 13.24 & Astrocytoma II & Drop-out \\
\hline РAT28 & M & 44 & 1.00 & Left & Frontal & 11.49 & Oligodendroglioma II & 245 \\
\hline РАT29 & M & 45 & 0.90 & Left & Frontal & 36.20 & Oligodendroglioma III & End of study \\
\hline РAT31 & $\mathrm{F}$ & 31 & 0.90 & Left & Frontal & 4.24 & Oligodendroglioma II & End of study \\
\hline
\end{tabular}

${ }^{1}$ Assessed before tumor resection; ${ }^{2}$-1=left-handed, $0=$ ambidextrous, $+1=$ right-handed; ${ }^{3}$ (Oligo-astrocytoma, ependymoma, and oligodendroglioma are subtypes of glioma tumors.

(more details are available in Aerts et al., 2018). Structural connectivity (SC) matrices were then constructed by transforming each individual's FreeSurfer parcellation scheme to the diffusion MRI data and calculating the number of estimated streamlines between each pair of brain regions. Lastly, we thresholded the SC matrices and normalized structural connections with the same constant scalar across subjects $(75,000$ in our case: 7.5 million streamlines generated per subject/100) as in the pre-operative analyses, to ensure all weights varied between 0 and 1 and were maximally comparable between pre- and post-operative assessment. 


\subsection{Brain network modeling}

Procedures for simulating large-scale brain dynamics and optimizing model parameters were also identical to those applied to the preoperative data, as described in detail in Aerts et al. (2018). Briefly, local dynamics for each of the 68 cortical brain regions were simulated using Reduced Wong-Wang neural mass models (Deco et al., 2014), which provides a satisfying approximation of the mean dynamics of interacting populations of excitatory and inhibitory spiking neurons. Subsequently, neural mass models were coupled according to each subject's tractography-derived structural connectome to generate personalized virtual brain models (Deco et al., 2014; Ritter et al., 2013; Sanz Leon et al., 2013; Schirner et al., 2018).

To optimize the correspondence between empirical and simulated functional connectivity, subject-specific parameter space explorations were conducted in which the global scaling parameter (G) was varied (0.01-3 in steps of 0.015). This parameter rescales each subject's structural connectivity, which is given by relative values, to yield absolute interaction strengths. For each parameter set, resting-state blood-oxygenlevel-dependent (BOLD) time series were generated. Subsequently, functional connectivity matrices were computed by calculating the Fisher's z-transformed Pearson correlation coefficient between all pairs of simulated BOLD time series. The parameter set that maximized the Pearson correlation between each individual's simulated and empirical functional connectivity matrix was then selected for further analyses.

In addition, inhibitory synaptic weights $\left(\mathrm{J}_{\mathrm{i}}\right)$ - which control the strength of connections from inhibitory to excitatory mass models within each large-scale region $i$ - were automatically tuned in each iteration of the parameter space exploration, to clamp the average firing rate at $3 \mathrm{~Hz}$ for each excitatory mass model (Deco et al., 2014; Schirner et al., 2018). After simulations, the obtained local inhibitory connection strengths were corrected for their respective region size (region size was regressed out from the values) for further analyses, since the need for local inhibition to balance global excitation depends on the total connection strength a brain region has, which tightly correlates with region size (Aerts et al., 2018). Median $\mathrm{J}_{\mathrm{i}}$ values (both corrected for region size as well as uncorrected) across the entire brain and across tumor and non-tumor regions in brain tumor patients were then computed per subject. Of note, delineation of tumor and non-tumor regions was based on the pre-operative data, according to the following criteria (Aerts et al. (2018)). In glioma patients, tumor regions were defined as those cortical areas of the individual FreeSurfer parcellation that showed at least partial (i.e., minimum 1 voxel) overlap with the tumor mask. In meningioma patients, tumor regions consisted of regions that were (at least partially) displaced because of the tumor's mass effect. To estimate which regions were displaced by the meningioma, patients' anatomic images were transformed to MNI space (using FSL FLIRT with 12 DOF), and this transformation was applied to their tumor mask. Then, the overlap between subjects' tumor mask in MNI space and the fsaverage Desikan-Killiany atlas (Desikan et al., 2006) in MNI space was calculated. Parcels that showed at least 1 voxel overlap with the tumor mask were denoted tumor nodes.

\subsection{Graph analysis}

Post-operative structural network topology was assessed with various graph theory metrics of integration (global efficiency, communicability), segregation (clustering coefficient, local efficiency, modularity), and centrality (degree, strength, betweenness centrality, participation coefficient), as well as graph density, using the Brain Connectivity Toolbox (Rubinov and Sporns, 2010). After inspecting the relationships among these graph theory metrics by linear correlation and principal component analysis, three distinct graph theory metrics were retained $(|\mathrm{r}|<0.80$ and graph metrics separable by the first 2 principal components) for further analyses: global efficiency, modularity, and participation coefficient. The two latter measures $h$ were both calculated using the same modular decomposition, which was identified through modularity maximization across 100 iterations. The number of nodes corresponds to the number of parcels, and is then the same for all the subjects.

\subsection{Neuropsychological testing}

Cognitive performance of all participants was re-assessed after each patient's tumor resection using the Cambridge Neuropsychological Test Automated Battery (CANTAB ${ }^{\circledR}$; Cambridge Cognition (2017); All rights reserved; http://www.cambridgecognition.com). The same cognitive tasks were administered as before surgery, again in random order to avoid sequence bias. In particular, the Rapid Visual Information Processing (RVP) task was used to assess sustained attention, the Spatial Span (SSP) task measured working memory capacity, the Reaction Time task (RTI) evaluated mental response speed, and the Stockings of Cambridge (SOC) task assessed planning accuracy.

\subsection{Accounting for covariates}

Several factors can influence cognitive performance and graph metrics (see for example Bettus et al., 2010; Biswal et al., 2010; Harrison et al., 2008). Therefore, cognitive performance results were corrected for each participant's level of emotional distress, residual lesion size, age and sex. Likewise, graph metrics were corrected for each subject's level of emotional distress, residual lesion size, age, sex, handedness, motion during resting-state fMRI acquisition and intensity normalization factor used in dMRI preprocessing. In particular, on the day testing took place, emotional distress was measured using the State-Trait Anxiety Inventory (Spielberger et al., 1983; Van der Ploeg, 1982). Further, residual lesion volume was calculated as the number of $1 \mathrm{~mm}^{3}$ isotropic voxels in the mask that delineated residual lesion tissue, which was drawn manually on the anatomical T1-weighted MRI image. Lastly, handedness was measured using the Edinburgh Handedness Inventory (Oldfield, 1971).

We then constructed linear regression models for every outcome variable (sustained attention, working memory capacity, reaction time, and planning accuracy for cognitive performance; global efficiency, modularity and participation coefficient for graph theory metrics) as a function of these confounders. Residuals of these models were further transformed to z-scores for subsequent analyses using the pre-operative mean and standard deviation of the respective metric in the group of control subjects, for ease of interpretation.

\subsection{Statistical analyses}

First, we compared post-operatively optimized model parameters, cognitive performance scores and graph metrics between glioma patients, meningioma patients and control participants. For these analyses, we used measures of association which are robust to the presence of outliers, and suitable for multiple comparisons. We used the implementations contained in the robust statistical toolbox by Rand Wilcox (Rallfun-v37.txt, update September 2019), using R version 3.5.3 (R Core Team, 2018). For measures of association we use the skipped Spearman correlation with adjusted p-values in conjunction with Hochberg's method to control FWE. This approach is referred to as L3 in Wilcox et al. (2018), and implemented in the R function scorregciH.

Pairs of groups are compared with an approach using 20\% trimmed means and percentile bootstrap (Wilcox and Rousselet, 2018), R function linconpb. Difference scores between pre- and post-operative quantities of interests across all participants were also tested using 20\% trimmed means with percentile bootstrap (Wilcox and Rousselet, 2018), R function dtrimpb. For these two latter measures, results are reported in terms of an estimated value of the contrast $\Psi$ and a confidence interval for it, together with a $\mathrm{p}$ value. 


\subsection{Virtual tumor resection}

After examining the stability of fitted model parameters over time, this information was used to perform the first virtual neurosurgery analyses, to evaluate the potential of brain network modeling to predict brain dynamics after tumor resection. To this end, a procedure similar to the one described by Taylor and colleagues (Taylor et al., 2018) was adopted. In particular, each patient's actual surgery was mimicked by removing all streamlines from their pre-operative tractogram that intersect the resection mask that was retrospectively derived from the post-operative anatomical MRI data. Since standard tractography algorithms are currently unable to reliably reconstruct white matter streamlines within or in close proximity to tumorous tissue, a dedicated pipeline was developed for this second part of the study. This was of crucial importance to allow simulation of tumor resection procedures, since white matter tracts in the vicinity of the tumor have the highest probability of being removed during neurosurgery. These proof of concept analyses were performed on all glioma patients for which both pre- and post-operative data were available. Virtual neurosurgery was not performed on data from meningioma patients, as these tumors generally do not infiltrate healthy brain tissue and therefore are not represented within the tractogram or structural connectivity matrix. Fig. 2 illustrates the procedure used to predict post-surgical brain dynamics after virtual tumor resection, using brain network modeling.

\subsubsection{Reconstruction of pre-operative tumor structural connectome}

Starting from the preprocessed pre-operative dMRI data, a novel technique to model the diffusion signal was applied, named single-shell 3-tissue constrained spherical deconvolution (SS3T-CSD) (Dhollander and Connelly, 2016; Dhollander et al., 2016), using MRtrix3Tissue (https:
//3Tissue.github.io), a fork of the MRtrix3 software (Tournier et al., 2019). Investigating the performance of this technique for this purpose, we have recently shown that SS3T-CSD allows to reconstruct white matter streamlines within infiltrative tumors and in immediately adjacent tissue (Aerts et al., 2019).

Based on the resulting white matter fiber orientation distributions (FODs), probabilistic streamlines tractography was performed, using an FOD amplitude threshold of 0.07 (Tournier et al., 2010). Although white matter FODs could be estimated using SS3T-CSD within regions infiltrated by a tumor (Aerts et al., 2019), resulting white matter FOD amplitudes were substantially smaller in tumor regions compared to the rest of the brain. While this likely reflects the smaller portion of space taken up by axons (due to infiltrating tumor tissue) and/or damage to white matter tracts, it does pose a practical challenge to tractography algorithms, which rely on the aforementioned amplitude threshold to determine where and how far tractography may proceed. As detailed in Aerts et al. (2019), we overcame this by gradually reducing the FOD amplitude threshold close to and even more so within the tumor, based on its prior segmentation obtained registering the T1-weighted image to the diffusion data, further spatially smoothed using a Gaussian kernel with a standard deviation of $3 \mathrm{~mm}$ to introduce a smooth boundary extending slightly beyond - as well as within - the edges of the tumor. Anatomical constraints were also imposed to the generation of streamlines, informed by a segmented tissue image (Smith et al., 2012). Since this particular segmentation strategy misclassified tumorous tissue mostly as gray matter, which is then enforced to be an endpoint of white matter streamlines during tractography, a modified segmented tissue image was provided. Specifically, the tumor mask was filled with undamaged tissue from homologous regions within the contralateral hemisphere using a non-linear registration approach (Foulon et al., 2018;
A

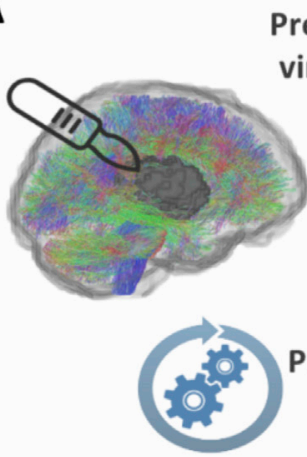
Pre-operative SC after
virtual neurosurgery

D

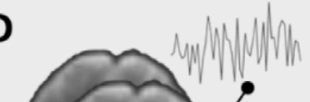

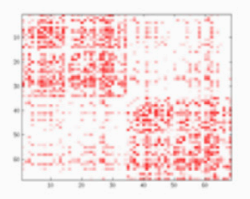

$+$

Pre-operatively fitted model parameters

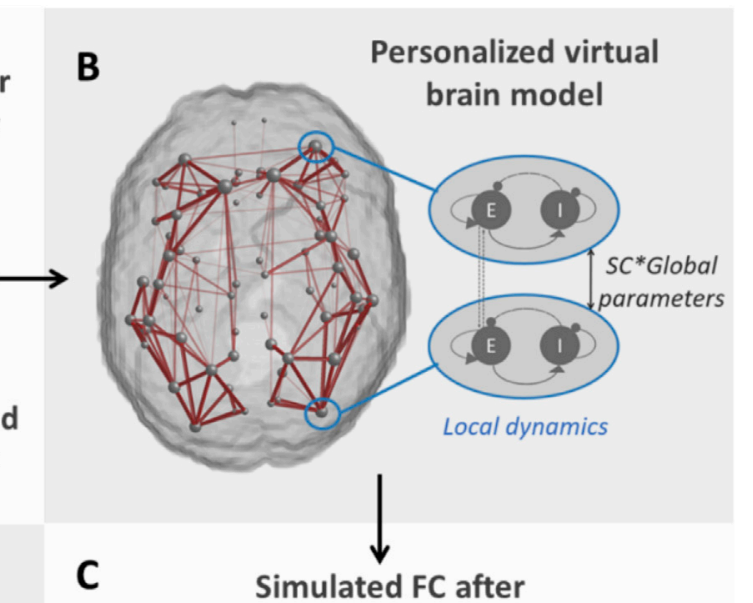

Post-operative empirical FC

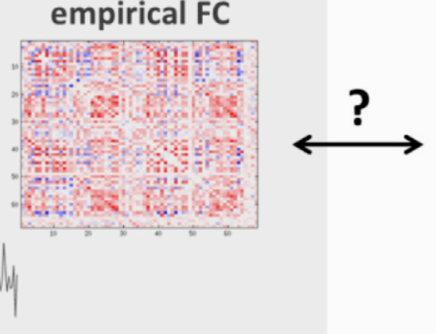

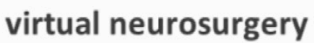

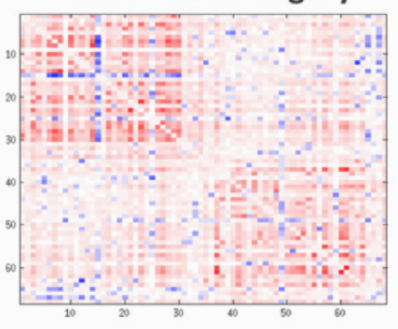

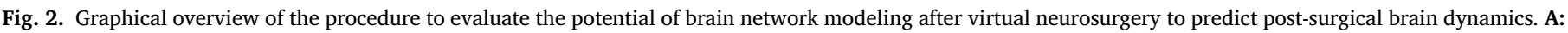

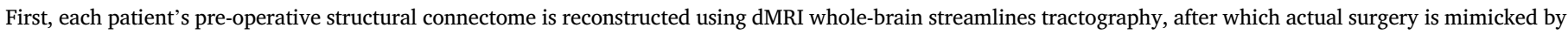

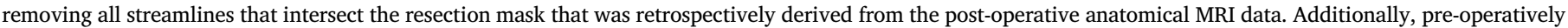

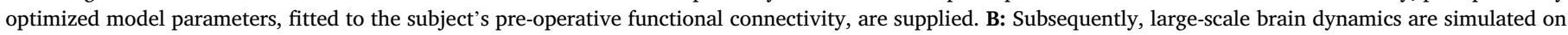

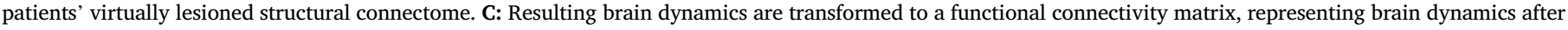

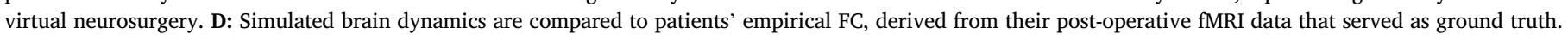


Nachev et al., 2008), providing an approximation of the patient's brain anatomy as if the tumor were absent. This "restored" anatomical image was then segmented and used to generate 30 million streamlines connecting pairs of brain regions.

All reconstructed streamlines were filtered to 7.5 million tracts using SIFT (Smith et al., 2013) to obtain quantitative streamline counts. Structural connectivity matrices were then constructed by calculating the number of estimated streamlines between any two Desikan-Killiany cortical brain regions, and normalizing all connectivity weights with a (single) constant scalar across subjects to ensure all weights varied between 0 and 1.

\subsubsection{Identifying optimal model parameters}

Using the newly constructed pre-surgical structural connectome, we redid subject-specific parameter space explorations to identify each patient's optimal global coupling value that maximized the correspondence between pre-surgical empirical and simulated functional connectivity. To this end, we adopted the procedure as described in section 2.3 ("Brain network modeling").

\subsubsection{Virtual lesioning of the structural connectome}

To mimic each patient's actual neurosurgical procedure retrospectively, tumor resection cavity maps were drawn manually under the supervision of an expert neuroradiologist (E.A.) based on the patient's postoperative anatomical T1-weighted MRI data. These resection masks were then overlaid onto the patient's pre-surgical tractogram using non-linear registration, and all connections that intersected the resection cavity mask were removed, similar to the approach outlined in Taylor et al. (2018).

\subsubsection{Simulating post-surgical brain dynamics}

Using the patient's pre-surgically optimized model parameters and virtually lesioned structural connectome, we then simulated large-scale brain dynamics with the Reduced Wong Wang model (Deco et al., 2014). Finally, each patient's predicted functional connectome was compared to their post-operative empirical functional connectome that served as ground truth, by means of link-wise Pearson correlation.

\subsection{Data and code accessibility}

All data and code used for this study is freely available. The data is publicly available at the OpenNeuro website (https://openneuro.org) and on the European Network for Brain Imaging of Tumors (ENBIT) repository (https://www.enbit.ac.uk) under the names "BTC_preop" and "BTC_postop" for the pre- and post-operative data, respectively. The optimized TVB C code can be found at https://github.com/BrainModes /The-Hybrid-Virtual-Brain and all scripts for postprocessing can be found at https://github.com/haerts/The-Virtual-Brain-Tumor-free-Pat ient.

\section{Results}

\subsection{Stability of individual model parameters over time}

In the first part of this study we examine if and how individually optimized model parameters change after tumor resection. In order to identify normal ranges of variability over time, we perform the same analyses in a group of healthy control subjects. Results are summarized in Table 2 and Fig. 3. In particular, the subplots on the left of Fig. 3 show the difference scores between pre- and post-operatively optimized model parameters in meningioma patients, glioma patients and control subjects. Differences over time in fitted parameters are shown as deviations from the horizontal line drawn around zero, with positive scores indicating increases in post-operative relative to pre-operative measures, whereas negative scores correspond to decreases after surgery compared with preoperative levels. The subplots on the right of Fig. 3 depict pre-versus post-
Table 2

Statistical results of analyses on differences between groups ( $\mathrm{C}=$ control, $\mathrm{M}=$ meningioma, $\mathrm{G}=$ glioma) and over time (pooling all subjects) of optimized model parameters. $\Psi$ represents the estimate of the contrast, followed by the confidence interval and the $\mathrm{p}$ value associated to it.

\begin{tabular}{|c|c|c|c|}
\hline & Pre-operative & Post-operative & $\begin{array}{l}\text { Difference scores } \\
\text { (post-pre) }\end{array}$ \\
\hline $\begin{array}{l}\mathbf{J}_{\text {tumor }} \\
\text { (corrected for ROI } \\
\text { size) }\end{array}$ & $\begin{array}{l}\text { C-M } \Psi=0.26 \\
{[0.090 .39], \mathrm{p}=} \\
0.00 \\
\mathrm{C}-\mathrm{G} \Psi=1.12 \\
{[0.021 .85], \mathrm{p}=} \\
0.00 \\
\mathrm{M}-\mathrm{G} \Psi=0.86 \\
{[0.051 .63], \mathrm{p}=} \\
0.01\end{array}$ & $\begin{array}{l}\text { C-M } \Psi=-0.26 \\
{[0.120 .52], \mathrm{p}=} \\
0.00 \\
\text { C-G } \Psi=1.08 \\
{[0.321 .76], \mathrm{p}=} \\
0.00 \\
\mathrm{M}-\mathrm{G} \Psi=0.82 \\
{[-0.021 .54], \mathrm{p}=} \\
0.02\end{array}$ & 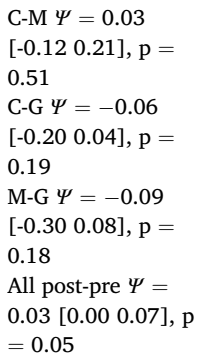 \\
\hline $\begin{array}{l}\mathbf{J}_{\text {non-tumor }} \\
\quad \text { (corrected for } \\
\text { ROI size) }\end{array}$ & $\begin{array}{l}\mathrm{C}-\mathrm{M} \Psi=-0.06 \\
{[-0.1-0.02], \mathrm{p}=} \\
0.00 \\
\mathrm{C}-\mathrm{G} \Psi=-0.02 \\
{[-0.070 .03], \mathrm{p}=} \\
0.31 \\
\mathrm{M}-\mathrm{G} \Psi=0.03 \\
{[-0.020 .10], \mathrm{p}=} \\
0.16\end{array}$ & $\begin{array}{l}\text { C-M } \Psi=-0.07 \\
{[-0.12-0.03], \mathrm{p}} \\
=0.00 \\
\mathrm{C}-\mathrm{G} \Psi=-0.01 \\
{[-0.060 .04], \mathrm{p}=} \\
0.67 \\
\mathrm{M}-\mathrm{G} \Psi=0.06 \\
{[0.010 .12], \mathrm{p}=} \\
0.01\end{array}$ & $\begin{array}{l}\text { C-M } \Psi=-0.03 \\
{[-0.080 .04], \mathrm{p}=} \\
0.31 \\
\mathrm{C}-\mathrm{G} \Psi=-0.00 \\
{[-0.040 .06], \mathrm{p}=} \\
0.70 \\
\mathrm{M}-\mathrm{G} \Psi=0.03 \\
{[-0.030 .09], \mathrm{p}=} \\
0.18 \\
\text { All post-pre } \Psi= \\
0.02[0.010 .04], \mathrm{p} \\
=0.01\end{array}$ \\
\hline $\begin{array}{l}\mathbf{J}_{\text {tumor }} \text { (uncorrected } \\
\text { for ROI size) }\end{array}$ & $\begin{array}{l}\text { C-M } \Psi=-0.07 \\
{[-0.410 .02], \mathrm{p}=} \\
0.08 \\
\text { C-G } \Psi=-0.34 \\
{[-0.58-0.07], \mathrm{p}} \\
=0.00 \\
\mathrm{M}-\mathrm{G} \Psi=-0.27 \\
{[-0.510 .12], \mathrm{p}=} \\
0.10\end{array}$ & $\begin{array}{l}\text { C-M } \Psi=-0.05 \\
{[-0.220 .11], \mathrm{p}=} \\
0.47 \\
\text { C-G } \Psi=-0.37 \\
{[-0.78-0.04], \mathrm{p}} \\
=0.01 \\
\mathrm{M}-\mathrm{G} \Psi=-0.32 \\
{[-0.750 .07], \mathrm{p}=} \\
0.05\end{array}$ & $\begin{array}{l}\text { C-M } \Psi=0.05 \\
{[-0.100 .29], \mathrm{p}=} \\
0.42 \\
\mathrm{C}-\mathrm{G} \Psi=-0.09 \\
{[-0.300 .17], \mathrm{p}=} \\
0.30 \\
\mathrm{M}-\mathrm{G} \Psi=-0.14 \\
{[-0.440 .13], \mathrm{p}=} \\
0.19 \\
\text { All post- pre } \Psi= \\
0.02[-0.030 .08], \mathrm{p} \\
=0.51\end{array}$ \\
\hline $\begin{array}{l}\mathbf{J}_{\text {non-tumor }} \\
\text { (uncorrected for } \\
\text { ROI size) }\end{array}$ & $\begin{array}{l}\text { C-M } \Psi=-0.00 \\
{[-0.050 .07], \mathrm{p}=} \\
0.97 \\
\text { C-G } \Psi=0.03 \\
{[-0.020 .10], \mathrm{p}=} \\
0.19 \\
\text { M-G } \Psi=0.03 \\
{[-0.020 .09], \mathrm{p}=} \\
0.15\end{array}$ & $\begin{array}{l}\text { C-M } \Psi=-0.02 \\
{[-0.100 .08], \mathrm{p}=} \\
0.57 \\
\text { C-G } \Psi=0.02 \\
{[-0.040 .11], \mathrm{p}=} \\
0.29 \\
\mathrm{M}-\mathrm{G} \Psi=0.05 \\
{[-0.050 .15], \mathrm{p}=} \\
0.25\end{array}$ & $\begin{array}{l}\text { C-M } \Psi=-0.03 \\
{[-0.090 .06], \mathrm{p}=} \\
0.42 \\
\text { C-G } \Psi=-0.01 \\
{[-0.090 .07], \mathrm{p}=} \\
0.71 \\
\mathrm{M}-\mathrm{G} \Psi=0.02 \\
{[-0.070 .09], \mathrm{p}=} \\
0.56 \\
\text { All post-pre } \Psi= \\
0.02[-0.010 .04], \mathrm{p} \\
=0.18\end{array}$ \\
\hline G & $\begin{array}{l}\text { C-M } \Psi=0.05 \\
{[-0.240 .30], \mathrm{p}=} \\
0.75 \\
\text { C-G } \Psi=0.23 \\
{[-0.100 .50], \mathrm{p}=} \\
0.09 \\
\text { M-G } \Psi=0.18 \\
{[-0.140 .49], \mathrm{p}=} \\
0.18\end{array}$ & $\begin{array}{l}\text { C-M } \Psi=-0.17 \\
{[-0.590 .21], \mathrm{p}=} \\
0.25 \\
\text { C-G } \Psi=0.01 \\
{[-0.310 .40], \mathrm{p}=} \\
0.95 \\
\text { M-G } \Psi=0.19 \\
{[-0.290 .60], \mathrm{p}=} \\
0.38\end{array}$ & 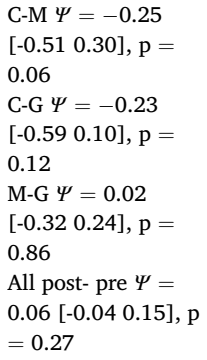 \\
\hline
\end{tabular}

operatively optimized model parameters at the individual level, shapeand color-coded by group. Here, differences over time in individuals' fitted parameters are shown as deviations from the main diagonal, with scores above the diagonal indicating increases in post-operative relative to pre-operative measures, whereas measures below the main diagonal correspond to decreases after surgery compared with pre-operative levels.

Specifically, Figs. $3 \mathrm{~A}$ and $2 \mathrm{~B}$ depict the median local inhibitory 

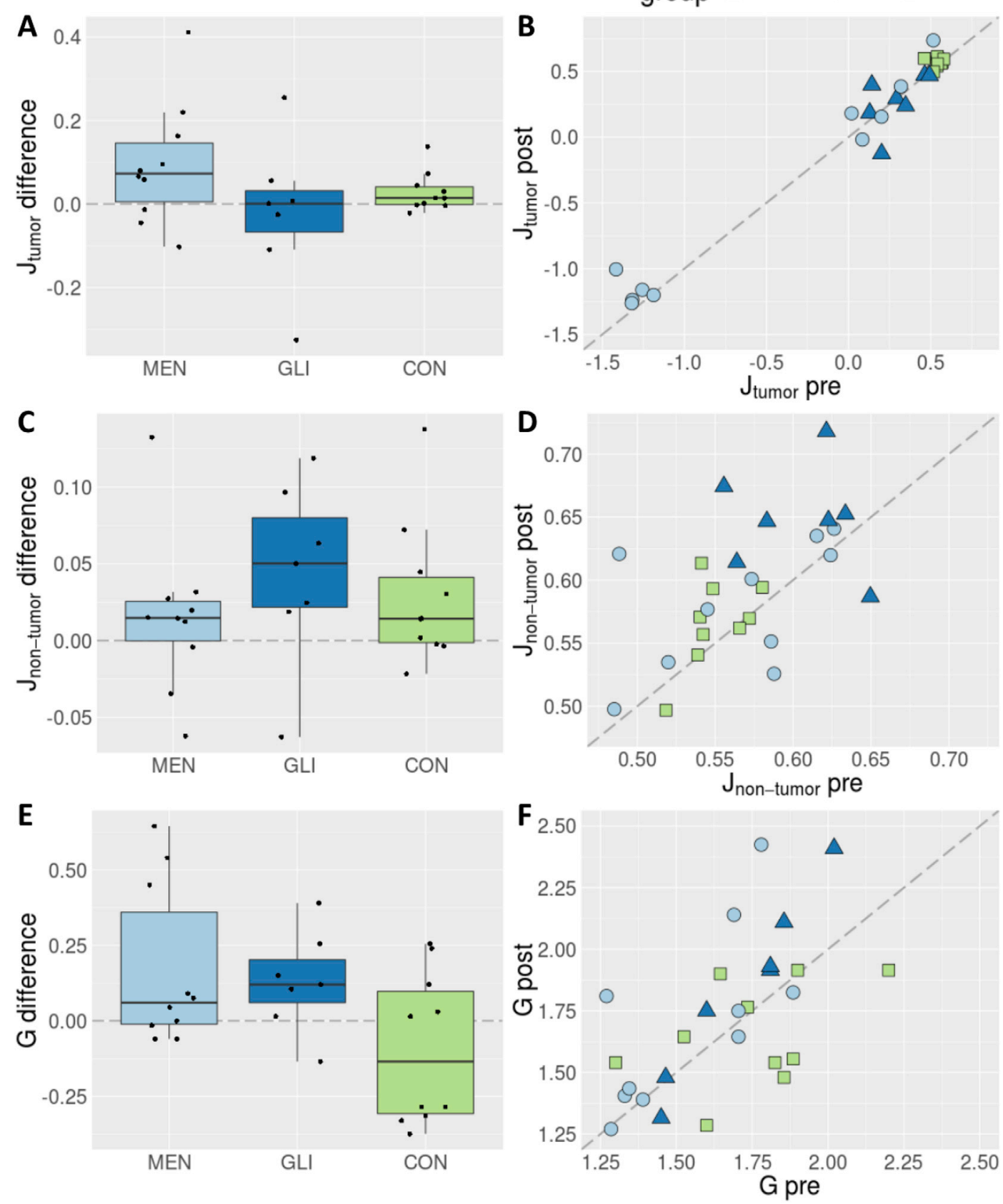

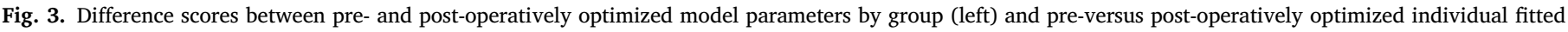

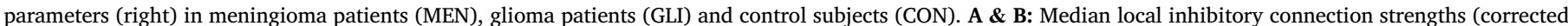

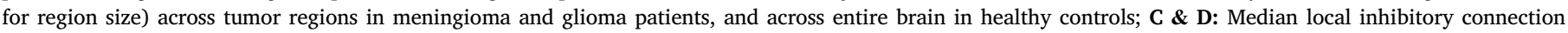

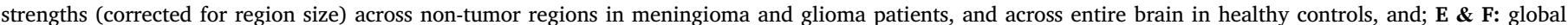
scaling parameter.

connection strengths across tumor regions in meningioma and glioma patients, and across the entire brain in healthy controls (after correcting for region size). Changes over time in local inhibitory connection strengths are not statistically significant, nor do they differ significantly between groups (Table 2), despite of the visual impression that local inhibitory connection strengths appear to be more variable in tumor regions compared to those in healthy brains, and displaying more negative values, before as well as after tumor resection.

Further, results reveal differences in median local inhibitory connection strengths (corrected for region size) across non-tumor regions in tumor patients relative to those in healthy brains (Table 2, Fig. 3C).
This is the case both before patients' tumor resection, as well as after surgery. Moreover, we observe an increase in participants' median local inhibitory connection strengths after surgery compared to their presurgical levels, although changes over time do not differ significantly between groups.

Of note, without correcting for region size, we also find significant differences in median local inhibitory connection strengths between glioma and healthy brain regions. However, without correction for region size, brain tumor patients show higher levels of feedback inhibition compared to controls, whereas the opposite trend was found when correcting for region size (see Supplementary Figs. 2A and 1B). Changes 
over time in local inhibitory connection strengths are also not statistically significant, and do not differ significantly between groups. In contrast, no significant differences are apparent in median local inhibitory connection strengths between non-tumor and healthy brain regions without correction for region size (Supplementary Figs. 2C and 1D).

Finally, we find no statistically significant group differences nor effects over time in the global scaling parameter (Supplementary Figs. 3E and $2 \mathrm{~F}$ ).

\subsection{Stability of structural network topology and cognitive performance over time}

Before relating optimized model parameters to cognitive performance scores and structural network topology metrics, we examine whether changes in these predictor variables can be observed over time, or whether post-operative group differences are apparent. Statistical results are summarized in Table 3, and Supplementary Figs. 3 and 3 provide a visual overview of pre- and post-operative cognitive performance and graph metrics (z-scores), respectively.

With regard to cognitive performance, results from prior work (Aerts et al., 2018) showed no statistically significant group differences before surgery in any of the cognitive domains assessed. Likewise, we find no

Table 3

Statistical results of analyses on differences over time (pooling all subjects) and between groups of cognitive performance and structural network topology scores. $\mathrm{C}=$ control, $\mathrm{M}=$ meningioma, $\mathrm{G}=$ glioma. $\Psi$ represents the estimate of the contrast, followed by the confidence interval and the $\mathrm{p}$ value associated to it.

\begin{tabular}{|c|c|c|}
\hline & Difference scores (post-pre) & $\begin{array}{l}\text { Post-operative group } \\
\text { differences }\end{array}$ \\
\hline Reaction time & $\begin{array}{l}\text { All post- pre } \Psi=-0.31[-0.88 \\
0.32], \mathrm{p}=0.3\end{array}$ & $\begin{array}{l}\mathrm{C}-\mathrm{M} \Psi=0.25[-2.452 .84] \\
\mathrm{p}=0.79 \\
\mathrm{C}-\mathrm{G} \Psi=-0.07[-1.32 \\
2.62], \mathrm{p}=0.84 \\
\mathrm{M}-\mathrm{G} \Psi=-0.18[-1.93 \\
2.60], \mathrm{p}=0.93\end{array}$ \\
\hline Sustained attention & $\begin{array}{l}\text { All post- pre } \Psi=0.13[-0.27 \\
0.48], \mathrm{p}=0.52\end{array}$ & $\begin{array}{l}\mathrm{C}-\mathrm{M} \Psi=-0.20[-1.41 \\
1.59], \mathrm{p}=0.84 \\
\mathrm{C}-\mathrm{G} \Psi=-0.10[-1.18 \\
1.13], \mathrm{p}=0.83 \\
\mathrm{M}-\mathrm{G} \Psi=0.09[-1.87 \\
1.59], \mathrm{p}=1.00\end{array}$ \\
\hline Planning accuracy & $\begin{array}{l}\text { All post- pre } \Psi=0.13[-0.35 \\
0.54], \mathrm{p}=0.6\end{array}$ & $\begin{array}{l}\mathrm{C}-\mathrm{M} \Psi=0.29[-0.871 .71] \\
\mathrm{p}=0.55 \\
\mathrm{C}-\mathrm{G} \Psi=0.05[-1.231 .28] \\
\mathrm{p}=0.93 \\
\mathrm{M}-\mathrm{G} \Psi=-0.24[-1.67 \\
0.91], \mathrm{p}=0.62\end{array}$ \\
\hline Working memory & $\begin{array}{l}\text { All post- pre } \Psi=0.01[-0.33 \\
0.40], \mathrm{p}=0.92\end{array}$ & $\begin{array}{l}\mathrm{C}-\mathrm{M} \Psi=0.25[-2.462 .84] \\
\mathrm{p}=0.79 \\
\mathrm{C}-\mathrm{G} \Psi=0.07[-1.42 \text { 2.62], } \\
\mathrm{p}=0.84 \\
\mathrm{M}-\mathrm{G} \Psi=-0.18[-1.93 \\
2.60], \mathrm{p}=0.93\end{array}$ \\
\hline Modularity & $\begin{array}{l}\text { All post- pre } \Psi=-0.05[-0.51 \\
0.40], \mathrm{p}=0.84\end{array}$ & $\begin{array}{l}\mathrm{C}-\mathrm{M} \Psi=0.34[-1.591 .84] \\
\mathrm{p}=0.67 \\
\mathrm{C}-\mathrm{G} \Psi=1.29[-0.782 .66] \\
\mathrm{p}=0.13 \\
\mathrm{M}-\mathrm{G} \Psi=0.95[-0.96 \\
2.67], \mathrm{p}=0.28\end{array}$ \\
\hline Global efficiency & $\begin{array}{l}\text { All post- pre } \Psi=0.85[-0.41 \\
0.59], \mathrm{p}=0.78\end{array}$ & $\begin{array}{l}\text { C-M } \Psi=-0.11[-1.58 \\
2.45], \mathrm{p}=0.94 \\
\text { C-G } \Psi=-0.82[-1.08 \\
3.02], \mathrm{p}=0.28 \\
\mathrm{M}-\mathrm{G} \Psi=0.93[-1.56 \\
2.16], \mathrm{p}=0.93\end{array}$ \\
\hline $\begin{array}{r}\text { Participation } \\
\text { coefficient }\end{array}$ & $\begin{array}{l}\text { All post- pre } \Psi=0.08[-0.75 \\
0.73], \mathrm{p}=0.91\end{array}$ & $\begin{array}{l}\mathrm{C}-\mathrm{M} \Psi=-0.81[-2.80 \\
1.47], \mathrm{p}=0.37 \\
\mathrm{C}-\mathrm{G} \Psi=-0.33[-3.04 \\
2.64], \mathrm{p}=0.88 \\
\mathrm{M}-\mathrm{G} \Psi=0.48[-1.95 \\
3.27], \mathrm{p}=0.61\end{array}$ \\
\hline
\end{tabular}

significant changes from pre-to post-operative assessment. Consequently, no group differences are apparent in cognitive performance after patients' tumor resection.

Concerning structural network topology, we find no significant changes over time. Furthermore, no statistically significant group differences are found in these post-operative graph metrics, despite increased levels of participation coefficient in glioma patients were observed before surgery (Aerts et al., 2018).

\subsection{Robust associations between modeling parameters, structural network topology and cognitive performance}

In the next step, we investigate the relations between the individually optimized modeling parameters on the one hand, and structural network topology and cognitive performance on the other hand.

A negative correlation between global efficiency of the structural network and the global scaling factor is replicated after patients' tumor resection, as shown in Fig. 4 ( $\rho=-.71, \mathrm{CI}=[-0.87-.40]$, p_adjusted $=$ 0.003).

The rest of the associations are not significative.

\subsection{Virtual neurosurgery proof of concept}

In order to evaluate the capacity of the currently applied brain network models to predict patients' post-surgical brain dynamics, we simulate brain dynamics after virtual neurosurgery and compare the resulting simulated functional connectivity to patients' empirical postoperative functional connectivity that served as ground truth. As a reference of how well the model can perform for a given patient, we also compute the maximum similarity between each patient's pre-operative empirical and simulated functional connectome performing parameter optimization, without virtual surgery.

Results of these proof of concept analyses are summarized in Fig. 5. Compared to the structural connectome that is used as input (SC), simulating functional connectivity by means of brain network modeling (FCsim) usually improves the correspondence with empirically derived functional connectivity (pre-op FCemp: pre-op FCsim > pre-op FCemp: pre-op SC) at the group level. Yet, important individual differences are evident in the extent to which computational modeling can enhance correlation with the empirical FC beyond the structural connectome. As can be seen in Fig. 5, substantial improvements in correlation with the empirical pre-op FC due to simulating brain activity optimizing parameter space exploration are observed in four out of seven patients (with respect to the distribution of the same quantity in all the subjects involved in the pre-operatory study), whereas the remaining three patients show little or no gain. This aspect - i.e., the degree to which the model can increase correlation with the empirical FC beyond the underlying structure - could play a role in assessing the potential of this technique for virtual neurosurgery (VS). In particular, prediction of postsurgical brain dynamics only improves in three out of four glioma patients for which computational modeling also yield substantially improved correlation with the empirical post-op FC beyond the structural connectome during parameter space exploration. In the other four patients, correspondence with empirical functional connectivity decreases after simulating virtual neurosurgery compared to using only the virtually lesioned structural connectivity matrix. With the present data we cannot say whether these aspects are correlated, and if the tumor size or location could make a difference.

\section{Discussion}

Results from our study reveal that model parameters describing brain dynamics are relatively stable over time in brain tumor patients who underwent tumor resection, relative to baseline variability levels observed in healthy control subjects. The association between global scaling parameter and efficiency of the structural connectivity appears to 


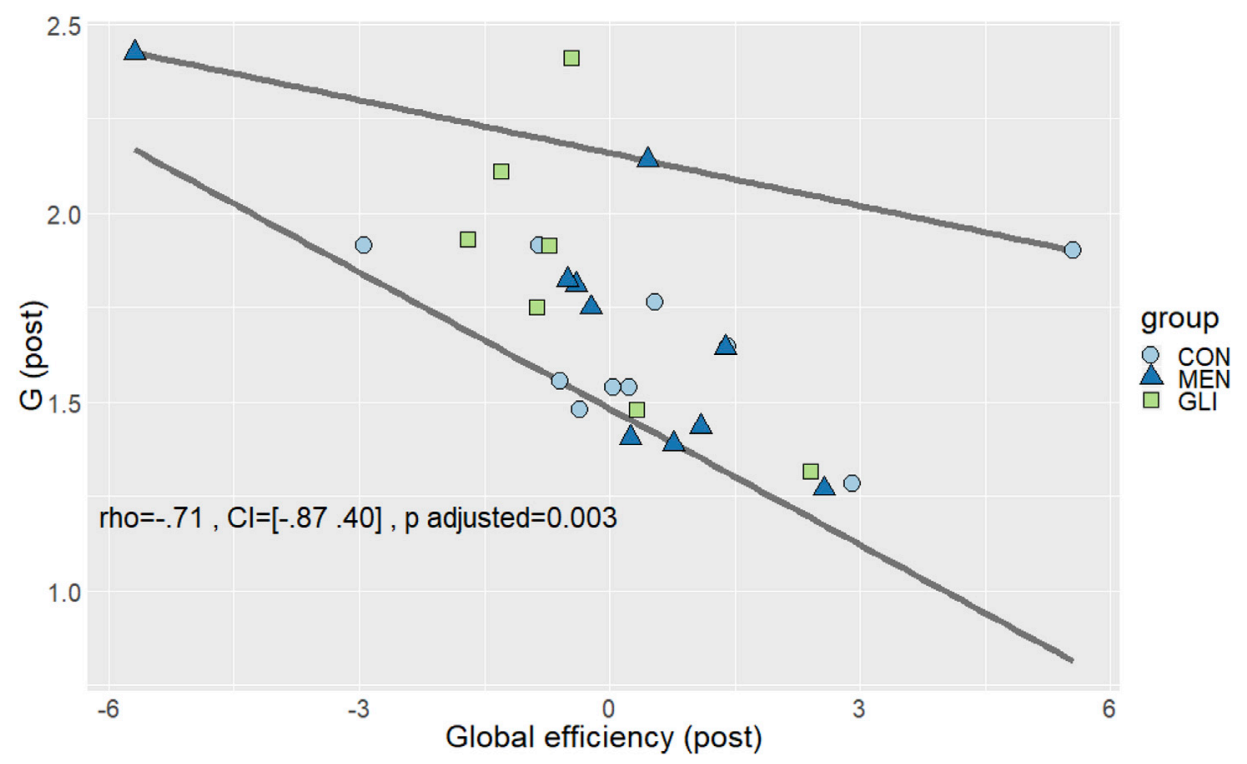

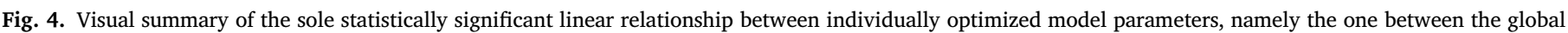

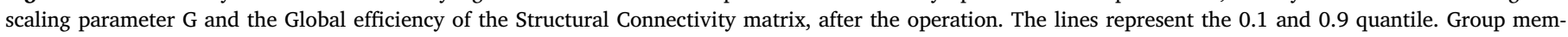
bership is shape- and color-coded: MEN = meningioma patients; GLI = glioma patients; CON = healthy controls.
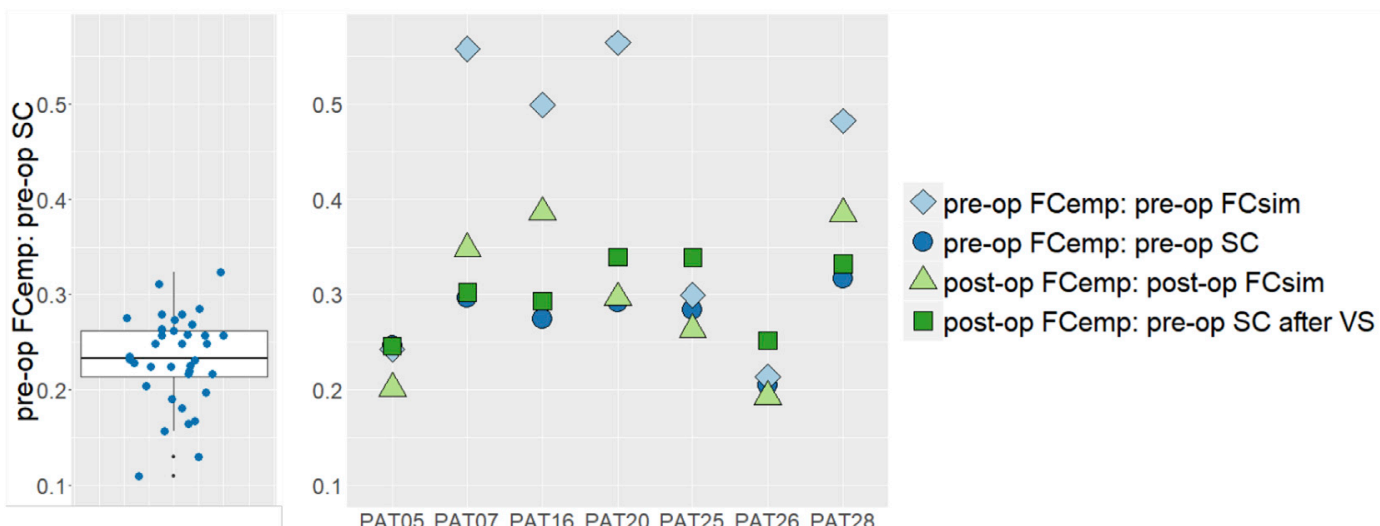

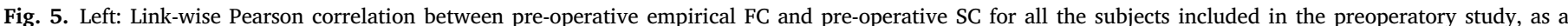

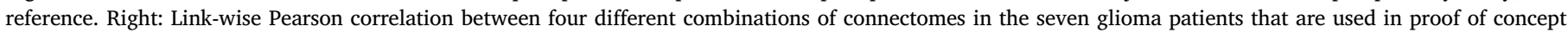

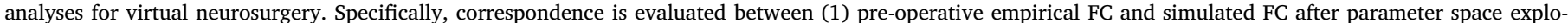

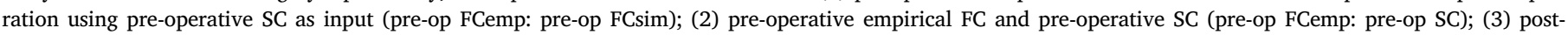

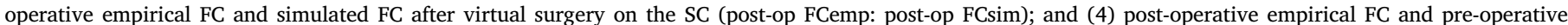
SC after virtual surgery (post-op FCemp: pre-op SC after VS).

be particularly robust both before and after surgery. Based on these findings, we perform the first proof of concept analyses to evaluate the potential of the currently applied brain network models to predict individual brain dynamics after tumor resection, relying solely on preoperatively available information. We obtain promising results for a subset of patients, and reveal several limitations and challenges that need to be addressed by future research.

\subsection{Individual biophysical model parameters and their predictors remain} stable over time

In contrast to our expectations, the amount of variability in individually optimized model parameters from pre-to post-operative assessment is comparable between brain tumor patients who underwent neurosurgery and healthy control subjects tested across a similar time interval. This means that there is some variability present over time in brain tumor patients' optimal model parameters, although there is no systematic trend towards increases or decreases in specific model parameters and the amount of variability does not exceed regular test-retest variability levels in healthy control subjects. Only inhibition control parameters across healthy regions are higher across all participants after surgery compared to pre-operative levels. Of note, we observe similar slight increases in feedback inhibition across tumor regions as well, although these differences do not reach statistical significance given the much larger variability of feedback inhibition values across tumor regions. These elevated levels in feedback inhibition could indicate that more inhibition was required to balance the marginally higher levels of global excitation (i.e., global coupling) after surgery.

In the next step, we evaluate structural network properties and measures of cognitive performance as possible predictors of the individual model parameters. In line with pre-operative results described in prior work (Aerts et al., 2018), initial descriptive analyses show remarkable similarity of cognitive performance and structural network topology across groups as well as over time. Only the increased 
participation coefficient that was found before surgery in glioma patients is no longer significant after tumor resection, pointing towards a normalization of glioma patients' structural network topology after surgery. While these findings seem counterintuitive, one other study has also reported comparable structural network topology in brain tumor patients relative to healthy controls ( $Y u$ et al., 2016). For cognitive performance, in contrast, the majority of previous studies have reported significant alterations in cognitive functioning as a result of a brain tumor or its subsequent treatment (Klein et al., 2002; Taphoorn and Klein, 2004; Taphoorn et al., 1994; Tucha et al., 2000). Possibly, the power of our analyses is not sufficient to detect differences between subjects or changes over time, given the limited sample size. Alternatively, the cognitive tasks (and graph metrics) that we utilize are not sufficiently sensitive to capture these differences.

Coming to the associations between cognitive performance and structural network topology with the individually optimized model parameters, we replicate the inverse relation between global efficiency of the structural network and the global scaling factor after patients' tumor resection. This implies that higher global coupling values are required in subjects whose structural connectome is less efficiently organized, in order to achieve the same amount of functional connectivity between cortical areas. This appears to be a very robust association, that has also been reported in stroke patients (Falcon et al., 2015). The positive relation between feedback inhibition across tumor regions in brain tumor patients and their reaction time during cognitive assessment is largely influenced by a few outlying observations, which would result in significant correlations assessed by some less robust approaches. Hence, caution is advised in interpreting this finding (and a larger sample size) would be required to clarify this association.

\subsection{Virtual neurosurgery results}

Given that brain tumor patients' model parameters remain relatively stable from pre-to post-operative assessment, we investigate whether post-operative brain dynamics can be predicted using only preoperatively available information. To this end, we virtually lesion the patient's pre-operative structural connectome according to the resection mask derived after surgery, based on which we re-simulate brain dynamics using a brain network model with the patients' pre-surgically optimized global coupling value. Any differences over time in feedback inhibition are assumed not to pose any problems for these proof of concept analyses, as the regional feedback inhibition control parameters are tuned automatically, to clamp the average firing rate at $3 \mathrm{~Hz}$ for each excitatory mass model.

The number of subjects that we could include in this analysis does not allow to clearly conclude that there is an improvement of the similarity between post-operative empirical FC and computational model implemented on virtually resected pre-operative SC. We found an indication that model performance during pre-operative parameter space exploration could be an indicator for the potential of this technique for virtual neurosurgery. In particular, prediction of post-surgical brain dynamics quantitatively increases in three out of four patients for which the model also substantially improves prediction accuracy beyond the structural connectome during parameter space exploration. In the fourth patient simulated post-operative brain dynamics do not show good correspondence with empirical post-operative brain dynamics; possibly the resection mask did not give an appropriate idea of the surgical intervention performed. In the other three patients for which computational modeling only results in marginal gains in prediction accuracy relative to the structural connectome, results show worse prediction accuracy after simulating post-operative brain dynamics compared to using only the virtually lesioned structural connectivity matrix. Nevertheless, for those patients, the virtually lesioned structural connectome serves as a good approximation of their post-operative functional connectivity, suggesting that their brain dynamics are more determined by the underlying structure in the months following tumor resection.

\subsection{Limitations and future directions}

In the interpretation of these results, some important limitations have to be taken into consideration. Our sample size is rather small, limiting the statistical power of the analyses. Additionally, substantial intersubject variability is present in both patient groups, caused by (among other factors) heterogeneity in lesion etiology, size and location. As a result, subtle differences between groups or over time are difficult to detect, and use of robust statistics is always recommended. By making use of increasingly available open-access clinical datasets, future studies may benefit from using larger sample sizes.

A larger sample size could also allow comparison of model parameters relative to tumor and non-tumor regions in patients with the same regions in healthy brains, since tumors have location preferences. This would further allow to control for region specificity of the parameters. Additionally, indicators currently used in neuro-oncology such as tumor grade, histology, lesion volume at diagnosis, isocitrate dehydrogenase (IDH)-status could be included as covariates in the prediction, the latter even as a model parameter.

Although feedback inhibition control parameters are controlled for region size, a substantial association between both remains. This may influence the results, since several tumors overlap with two very large regions (superiorfrontal left and right), whose inhibition values are much higher compared to those of other regions. Currently, model optimization processes are being improved in order to optimize regional feedback inhibition control parameters based on the empirical FC data rather than using the firing rate, which is a direct proxy of region size. Complementary, future research could use parcellation schemes with equally sized regions in order to avoid the confounding effect of region size.

Important individual differences are observed in the added benefit of simulating brain dynamics on top of the individual structural connectome. These results could reflect true differences in the appropriateness of the model between subjects. Alternatively, however, these differences may result from instabilities in the parameter optimization procedure by maximizing the link-wise Pearson correlation between empirical and simulated FC. Although this method is routinely employed in large-scale modeling studies, the use of robust statistics (Skipped spearman correlations for example) could lead to more reliable results. Furthermore, other methods that maximize the large-scale organization of both connectivity matrices might be explored. For example, similarity could be sought at the modular level, maximizing the cross-modularity between simulated and empirical functional connectivity (Diez et al., 2015; Stramaglia et al., 2017).

Also, computational model parameters can provide indications on actual neurobiological values that are normally not measurable in living humans, but any information not included in the model of the BOLD signal, such as blood perfusion, heart rate, respiration, personalized hemodynamics, will contribute to model uncertainty.

\section{Conclusion}

In summary, our study is the first investigation of potential changes in model parameters describing brain dynamics after brain tumor resection using large-scale brain network modeling. Notwithstanding the methodological caveats described above, we provide preliminary evidence that optimized model parameters are relatively stable from pre-to postoperative assessment. Based on these findings, we perform the first proof of concept analyses to evaluate the potential of brain network modeling to predict brain dynamics after tumor resection. We confirm that the approach is feasible and generalizable; on the other hand we reveal important limitations that need to be addressed by future research.

\section{CRediT authorship contribution statement}

Hannelore Aerts: Conceptualization, Methodology, Software, Validation, Formal analysis, Investigation, Data curation, Writing - original 
draft, Writing - review \& editing, Visualization. Michael Schirner: Software, Resources, Validation. Thijs Dhollander: Software, Resources, Validation. Ben Jeurissen: Software, Validation. Eric Achten: Investigation, Project administration, Resources, Project administration. Dirk Van Roost: Project administration, Funding acquisition. Petra Ritter: Conceptualization, Methodology, Software, Validation, Resources, Funding acquisition. Daniele Marinazzo: Conceptualization, Methodology, Software, Validation, Formal analysis, Investigation, Data curation, Writing - original draft, Writing - review \& editing, Visualization, Funding acquisition, Supervision.

\section{Acknowledgments}

This project has received funding from the Special Research Funds (BOF) of the University of Ghent (01MR0210 and 01J10715), Grant P7/ 11 from the Interuniversity Attraction Poles Program of the Belgian Federal Government, and the European Union's Horizon 2020 Framework Programme for Research and Innovation under the Specific Grant Agreement No. 785907 (Human Brain Project SGA2). T.D. acknowledges the National Health and Medical Research Council (NHMRC) of Australia and the Victorian Government's Operational Infrastructure Support Program for their support. P.R. acknowledges the following funding sources: H2020 Research and Innovation Action grants VirtualBrainCloud 826421 and Human Brain Project 785907 and ERC 683049; German Research Foundation CRC 1315 and grant RI 2073/6-1; Berlin Institute of Health \& Foundation Charité, Johanna Quandt Excellence Initiative. B.J. is a postdoctoral fellow supported by the Research Foundation Flanders (FWO Vlaanderen). Finally, we would like to thank Prof. Dr. Dirk Van Roost, Stephanie Bogaert, Robby De Pauw, Hannes Almgren, Iris Coppieters, Jeroen Kregel, Mireille Augustijn, Helena Verhelst and Kelly Berckmans for their help in acquiring the data.

\section{Appendix A. Supplementary data}

Supplementary data to this article can be found online at https://doi. org/10.1016/j.neuroimage.2020.116738.

\section{References}

Aerts, H., Dhollander, T., Marinazzo, D., 2019. Evaluating the performance of 3-tissue constrained spherical deconvolution pipelines for within-tumor tractography. BioRxiv. https://doi.org/10.1101/629873.

Aerts, H., Schirner, M., Jeurissen, B., Van Roost, D., Achten, E., Ritter, P., Marinazzo, D. 2018. Modeling brain dynamics in brain tumor patients using the virtual brain. ENeuro 5 (3). https://doi.org/10.1523/ENEURO.0083-18.2018.

Andersson, J.L.R., Jenkinson, M., Smith, S.M., 2007. Non-linear Registration, Aka Spatial Normalisation. FMRIB Technical Report TR07JA2. https://doi.org/10.1016/ j.neuroimage.2008.10.055.

Andersson, J.L.R., Skare, S., Ashburner, J., 2003. How to correct susceptibility distortions in spin-echo echo-planar images: application to diffusion tensor imaging. Neuroimage 20 (2), 870-888. https://doi.org/10.1016/S1053-8119(03)00336-7.

Andersson, J.L.R., Sotiropoulos, S.N., 2016. An integrated approach to correction for offresonance effects and subject movement in diffusion MR imaging. Neuroimage 125, 1063-1078. https://doi.org/10.1016/j.neuroimage.2015.10.019.

Arsiwalla, X.D., Zucca, R., Betella, A., Martinez, E., Dalmazzo, D., Omedas, P., et al., 2015 Network dynamics with Brain $\mathrm{X}^{3}$ : a large-scale simulation of the human brain network with real-time interaction. Front. Neuroinf. 9 https://doi.org/10.3389/ fninf.2015.00002, 02.

Bettus, G., Bartolomei, F., Confort-Gouny, S., Guedj, E., Chauvel, P., Cozzone, P.J., et al., 2010. Role of resting state functional connectivity MRI in presurgical investigation of mesial temporal lobe epilepsy. J. Neurol. Neurosurg. Psychiatry 81, 1147-1154. https://doi.org/10.1136/jnnp.2009.191460.

Biswal, B.B., Mennes, M., Zuo, X.-N., Gohel, S., Kelly, C., Smith, S.M., et al., 2010. Toward discovery science of human brain function. Proc. Natl. Acad. Sci. U.S.A. 107 (10), 4734-4739. https://doi.org/10.1073/pnas.0911855107.

Bonilha, L., Helpern, J.A., Sainju, R., Nesland, T., Edwards, J.C., Glazier, S.S., Tabesh, A., 2013. Presurgical connectome and postsurgical seizure control in temporal lobe epilepsy. Neurology 81, 1704-1710. https://doi.org/10.1212/ 01.wnl.0000435306.95271.5f.

Bonilha, L., Jensen, J.H., Baker, N., Breedlove, J., Nesland, T., Lin, J.J., et al., 2015. The brain connectome as a personalized biomarker of seizure outcomes after temporal lobectomy. Neurology 84, 1846-1853. https://doi.org/10.1212/ WNL.0000000000001548.
Deco, G., Ponce-Alvarez, A., Hagmann, P., Romani, G.L., Mantini, D., Corbetta, M., 2014. How local excitation - inhibition ratio impacts the whole brain dynamics. J. Neurosci. 34 (23), 7886-7898. https://doi.org/10.1523/JNEUROSCI.506813.2014.

Desikan, R.S., Ségonne, F., Fischl, B., Quinn, B.T., Dickerson, B.C., Blacker, D., et al., 2006. An automated labeling system for subdividing the human cerebral cortex on MRI scans into gyral based regions of interest. Neuroimage 31, 968-980. https:// doi.org/10.1016/j.neuroimage.2006.01.021.

Dhollander, T., Connelly, A., 2016. A novel iterative approach to reap the benefits of multi-tissue CSD from just single-shell $(+\mathrm{b}=0)$ diffusion MRI data. In: Proc. Intl. Soc. Mag. Reson. Med, vol. 24, p. 3010.

Dhollander, T., Raffelt, D., Connelly, A., 2016. Unsupervised 3-tissue response function estimation from single-shell or multi-shell diffusion MR data without co-registered T1 image. In: ISMRM Workshop on Breaking the Barriers of Diffusion MRI (P. 5).

Diez, I., Bonifazi, P., Escudero, I., Mateos, B., Muñoz, M.A., Stramaglia, S., Cortes, J.M., 2015. A novel brain partition highlights the modular skeleton shared by structure and function. Sci. Rep. 5, 10532. https://doi.org/10.1038/srep10532.

Doucet, G.E., Rider, R., Taylor, N., Skidmore, C., Sharan, A., Sperling, M., Tracy, J.I., 2015. Presurgery resting-state local graph-theory measures predict neurocognitive outcomes after brain surgery in temporal lobe epilepsy. Epilepsia 56 (4), 517-526. https://doi.org/10.1111/epi.12936.

Emblem, K.E., Nedregaard, B., Hald, J.K., Nome, T., Due-Tonnessen, P., Bjornerud, A., 2009. Automatic glioma characterization from dynamic susceptibility contrast imaging: brain tumor segmentation using knowledge-based fuzzy clustering. J. Magn. Reson. Imag. 30, 1-10. https://doi.org/10.1002/jmri.21815.

Emblem, K.E., Pinho, M.C., Zöllner, F.G., Due-Tonnessen, P., Hald, J.K., Schad, L.R., et al., 2015. A generic support vector machine model for preoperative glioma survival associations. Radiology 275 (1), 228-234. https://doi.org/10.1148/ radiol.14140770.

Falcon, M.I., Riley, J.D., Jirsa, V.K., McIntosh, A.R., Shereen, A.D., Chen, E.E., Solodkin, A., 2015. The Virtual Brain: modeling biological correlates of recovery after chronic stroke. Front. Neurol. 6, 228. https://doi.org/10.3389/fneur.2015.00228.

Fischl, B., van der Kouwe, A., Destrieux, C., Halgren, E., Ségonne, F., Salat, D.H., et al., 2004. Automatically parcellating the human cerebral cortex. Cerebr. Cortex 14, 11-22. https://doi.org/10.1093/cercor/bhg087.

Fisher, J.L., Schwartzbaum, J.A., Wrensch, M., Wiemels, J.L., 2007. Epidemiology of brain tumors. Neurol. Clin. 25, 867-890. https://doi.org/10.1016/j.ncl.2007.07.002.

Foulon, C., Cerliani, L., Kinkingnehun, S., Levy, R., Rosso, C., Urbanski, M., et al., 2018. Advanced Lesion Symptom Mapping Analyses and Implementation as BCBtoolkit. GigaScience. https://doi.org/10.1101/133314 giy004.

Goebel, S., Von Harscher, M., Mehdorn, H.M., 2011. Comorbid mental disorders and psychosocial distress in patients with brain tumours and their spouses in the early treatment phase. Support. Care Canc. 19 (11), 1797-1805. https://doi.org/10.1007/ s00520-010-1021-8.

Harrison, B.J., Pujol, J., Ortiz, H., Fornito, A., Pantelis, C., Yücel, M., 2008. Modulation of brain resting-state networks by sad mood induction. PloS One 3 (3), e1794. https:// doi.org/10.1371/journal.pone.0001794.

He, X., Doucet, G.E., Pustina, D., Sperling, M.R., Sharan, A.D., Tracy, J.I., 2017. Presurgical thalamic "hubness" predicts surgical outcome in temporal lobe epilepsy. Neurology 88, 1-9. https://doi.org/10.1212/WNL.0000000000004035.

Janda, M., Steginga, S., Langbecker, D., Dunn, J., Walker, D.G., Eakin, E., 2007. Quality of life among patients with a brain tumor and their carers. J. Psychosom. Res. 63, 617-623. https://doi.org/10.1016/j.jpsychores.2007.06.018.

Jenkinson, M., Bannister, P., Brady, M., Smith, S.M., 2002. Improved optimization for the robust and accurate linear registration and motion correction of brain images. Neuroimage 17, 825-841. https://doi.org/10.1016/S1053-8119(02)91132-8.

Jenkinson, M., Beckmann, C.F., Behrens, T.E.J., Woolrich, M.W., Smith, S.M., 2012. FSL. NeuroImage 62 (2), 782-790. https://doi.org/10.1016/j.neuroimage.2011.09.015.

Jenkinson, M., Smith, S.M., 2001. A global optimisation method for robust affine registration of brain images. Med. Image Anal. 5, 143-156. https://doi.org/10.1016/ S1361-8415(01)00036-6.

Jeurissen, B., Tournier, J.-D., Dhollander, T., Connelly, A., Sijbers, J., 2014. Multi-tissue constrained spherical deconvolution for improved analysis of multi-shell diffusion MRI data. Neuroimage 103, 411-426. https://doi.org/10.1016/ j.neuroimage.2014.07.061.

Ji, G.-J., Zhang, Z., Xu, Q., Wei, W., Wang, J., Wang, Z., et al., 2015. Connectome reorganization associated with surgical outcome in temporal lobe epilepsy. Medicine 94 (40), e1737. https://doi.org/10.1097/MD.0000000000001737.

Kellner, E., Dhital, B., Kiselev, V.G., Reisert, M., 2016. Gibbs-ringing artifact removal based on local subvoxel-shifts. Magn. Reson. Med. 76, 1574-1581. https://doi.org/ $10.1002 / \mathrm{mrm} .26054$.

Klein, M., Heimans, J.J., Aaronson, N.K., Van der Ploeg, H.M., Grit, J., Muller, M., et al., 2002. Effect of radiotherapy and other treatment-related factors on mid-term to longterm cognitive sequelae in low-grade gliomas: a comparative study. Lancet 360 (9343), 1361-1368. https://doi.org/10.1016/S0140-6736(02)11398-5.

Louis, D.N., Ohgaki, H., Wiestler, O.D., Cavenee, W.K., Burger, P.C., Jouvet, A., et al., 2007. The 2007 WHO classification of tumours of the central nervous system. Acta Neuropathol. 114 (2), 97-109. https://doi.org/10.1007/s00401-007-0243-4.

Morgan, V.L., Englot, D.J., Rogers, B.P., Landman, B.A., Cakir, A., Abou-Khalil, B.W., Anderson, A.W., 2017. Magnetic resonance imaging connectivity for the prediction of seizure outcome in temporal lobe epilepsy. Epilepsia 58 (7), 1251-1260. https:// doi.org/10.1111/epi.13762.

Munsell, B.C., Wee, C.-Y., Keller, S.S., Weber, B., Elger, C., Tomaz Da Silva, L.A., et al., 2015. Evaluation of machine learning algorithms for treatment outcome prediction in patients with epilepsy based on structural connectome data. Neuroimage 118, 219-230. https://doi.org/10.1016/j.neuroimage.2015.06.008. 
Nachev, P., Coulthard, E., Jäger, H.R., Kennard, C., Husain, M., 2008. Enantiomorphic normalization of focally lesioned brains. Neuroimage 39, 1215-1226. https:// doi.org/10.1016/j.neuroimage.2007.10.002.

Oldfield, R.C., 1971. The assessment and analysis of handedness: the Edinburgh inventory. Neuropsychologia 9 (1), 97-113.

Olmi, S., Petkoski, S., Guye, M., Bartolomei, F., Jirsa, V.K., 2019. Controlling seizure propagation in large-scale brain networks. PLoS Comput. Biol. 15 (2), e1006805 https://doi.org/10.1371/journal.pcbi.1006805.

Proix, T., Bartolomei, F., Guye, M., Jirsa, V.K., 2017. Individual brain structure and modelling predict seizure propagation. Brain 140, 641-654. https://doi.org/ 10.1093/brain/awx004.

R Core Team, 2018. R: a language and environment for statistical computing. Retrieved from. https://www.r-project.org/.

Ritter, P., Schirner, M., McIntosh, A.R., Jirsa, V.K., 2013. The virtual brain integrates computational modeling and multimodal neuroimaging. Brain Connect. 3 (2), 121-145. https://doi.org/10.1089/brain.2012.0120.

Rubinov, M., Sporns, O., 2010. Complex network measures of brain connectivity: uses and interpretations. Neuroimage 52 (3), 1059-1069. https://doi.org/10.1016/ j.neuroimage.2009.10.003.

Rughani, A.I., Dumont, T.M., Lu, Z., Bongard, J., Horgan, M.A., Penar, P.L., Tranmer, B.I., 2010. Use of an artificial neural network to predict head injury outcome. J. Neurosurg. 113 (3), 585-590. https://doi.org/10.3171/2009.11.JNS09857.

Sanz Leon, P., Knock, S.A., Woodman, M.M., Domide, L., Mersmann, J., McIntosh, A.R., Jirsa, V.K., 2013. The Virtual Brain: a simulator of primate brain network dynamics. Front. Neuroinf. 7, 10. https://doi.org/10.3389/fninf.2013.00010.

Schirner, M., McIntosh, A.R., Jirsa, V.K., Deco, G., Ritter, P., 2018. Inferring multi-scale neural mechanisms with brain network modelling. ELife 7, e28927. https://doi.org/ 10.7554/eLife.28927.

Senders, J.T., Arnaout, O., Karhade, A.V., Dasenbrock, H.H., Gormley, W.B., Broekman, M.L., Smith, T.R., 2017. Natural and artificial intelligence in neurosurgery: a systematic review. Neurosurgery 1-12. https://doi.org/10.1093/ neuros/nyx384, 0(0).

Senders, J.T., Staples, P.C., Karhade, A.V., Zaki, M.M., Gormley, W.B., Broekman, M.L.D. et al., 2018. Machine learning and neurosurgical outcome prediction: a systematic review. World Neurosurgery 109, 476-486. https://doi.org/10.1016/ j.wneu.2017.09.149.

Sinha, N., Dauwels, J., Kaiser, M., Cash, S.S., Westover, M.B., Wang, Y., Taylor, P.N., 2017. Predicting neurosurgical outcomes in focal epilepsy patients using computational modelling. Brain 140, 319-332. https://doi.org/10.1093/aww332.

Smith, R.E., Tournier, J.-D., Calamante, F., Connelly, A., 2012. Anatomically-constrained tractography: improved diffusion MRI streamlines tractography through effective use of anatomical information. Neuroimage 62 (3), 1924-1938. https://doi.org/ 10.1016/j.neuroimage.2012.06.005.

Smith, R.E., Tournier, J.-D., Calamante, F., Connelly, A., 2013. SIFT: sphericaldeconvolution informed filtering of tractograms. Neuroimage 67, 298-312. https:// doi.org/10.1016/j.neuroimage.2012.11.049.
Solodkin, A., Hasson, U., Siugzdaite, R., Schiel, M., Chen, E.E., Kotter, R., Small, S.L., 2010. Virtual Brain Transplantation (VBT): a method for accurate image registration and parcellation in large cortical stroke. Arch. Ital. Biol. 148, 219-241.

Spielberger, C.D., Gorsuch, R.L., Lushene, R., Vagg, P.R., Jacobs, G.A., 1983. Manual for the State-Trait Anxiety Inventory. Consulting Psychologists Press, Palo Alto, CA.

Stramaglia, S., Pellicoro, M., Angelini, L., Amico, E., Aerts, H., Cortés, J.M., et al., 2017. Ising model with conserved magnetization on the human connectome: implications on the relation structure-function in wakefulness and anesthesia. Chaos 27 (4), 047407. https://doi.org/10.1063/1.4978999.

Taphoorn, M.J.B., Klein, M., 2004. Cognitive deficits in adult patients with brain tumours. Lancet Neurol. 3, 159-168. https://doi.org/10.1016/S1474-4422(04)00680-5.

Taphoorn, M.J.B., Schiphorst, A.K., Snoek, F.J., Lindeboom, J., Wolbers, J.G., Karim, A.B.M.F., et al., 1994. Cognitive functions and quality of life in patients with low-grade gliomas: the impact of radiotherapy. Ann. Neurol. 36, 48-54.

Taylor, P.N., Sinha, N., Wang, Y., Vos, S.B., de Tisi, J., Miserocchi, A., et al., 2018. The impact of epilepsy surgery on the structural connectome and its relation to outcome. Neuroimage: Clinical 18, 202-214. https://doi.org/10.1016/j.nicl.2018.01.028.

Tournier, J.-D., Calamante, F., Connelly, A., 2010. Improved probabilistic streamlines tractography by 2 nd order integration over fibre orientation distributions. In: Proc. Intl. Soc. Mag. Reson. Med, vol. 18, p. 1670.

Tournier, J.-D., Smith, R.E., Raffelt, D., Tabbara, R., Dhollander, T., Pietsch, M., et al., 2019. MRtrix3: a fast, flexible and open software framework for medical image processing and visualisation. BioRxiv. https://doi.org/10.1101/551739.

Tucha, O., Smely, C., Preier, M., Lange, K.W., 2000. Cognitive deficits before treatment among patients with brain tumors. Neurosurgery 47 (2), 324-333.

van Dellen, E., Douw, L., Hillebrand, A., de Witt Hamer, P.C., Baayen, J.C., Heimans, J.J., et al., 2014. Epilepsy surgery outcome and functional network alterations in longitudinal MEG: a minimum spanning tree analysis. Neuroimage 86, 354-363. https://doi.org/10.1016/j.neuroimage.2013.10.010.

Van der Ploeg, H.M., 1982. De zelf-beoordelings vragenlijst angst. Tijdschr Psychiatr. 24, 576-588. Retrieved from. http://tijdschriftvoorpsychiatrie.be/issues/368/articles /2438.

Veraart, J., Novikov, D.S., Christiaens, D., Ades-aron, B., Sijbers, J., Fieremans, E., 2016. Denoising of diffusion MRI using random matrix theory. Neuroimage 142, 394-406. https://doi.org/10.1016/j.neuroimage.2016.08.016.

Wilcox, R.R., Rousselet, G.A., 2018. A guide to robust statistical methods in neuroscience. Current Protocols in Neuroscience 82 (1). https://doi.org/10.1002/cpns.41.

Wilcox, R.R., Rousselet, G.A., Pernet, C.R., 2018. Improved methods for making inferences about multiple skipped correlations. J. Stat. Comput. Simulat. 88 (16), 3116-3131. https://doi.org/10.1080/00949655.2018.1501051.

Yu, Z., Tao, L., Qian, Z., Wu, J., Liu, H., Yu, Y., et al., 2016. Altered brain anatomical networks and disturbed connection density in brain tumor patients revealed by diffusion tensor tractography. Int. J. Comput. Assist.Radiol.Surg 11 (11), 2007-2019. https://doi.org/10.1007/s11548-015-1330-y.

Zhang, Y., Brady, M., Smith, S.M., 2001. Segmentation of brain MR images through a hidden Markov random field model and the expectation-maximization algorithm. IEEE Trans. Med. Imag. 20 (1), 45-57. https://doi.org/10.1109/42.906424. 